\title{
ANÁLISIS PALEOPARASITOLÓGICO DE LA MUSCULATURA ESQUELÉTICA DE LA MOMIA DEL CERRO EL PLOMO, CHILE: TRICHINELLA SP.*
}

\author{
PALEOPARASITOLOGICAL ANALYSIS OF SKELETAL MUSCLE TISSUE \\ OF THE CERRO EL PLOMO MUMMY FROM CHILE: TRICHINELLA SP.
}

\author{
Héctor Rodríguez ${ }^{*}$, Isabel Noemi², José Luis Cerva², Omar Espinoza-Navarro ${ }^{3}$, \\ María Eugenia Castro $^{1}$ y Mario Castro ${ }^{4}$
}

\begin{abstract}
La momia del Cerro El Plomo es un niño Inca de 8 a 9 años de edad, que por datación relativa pertenecería al periodo del año 1500 aproximadamente. Esta momia fue encontrada por arrieros en las proximidades de la cima del Cerro El Plomo, frente a la ciudad de Santiago el año 1954, y desde entonces se encuentra depositada en el Museo Nacional de Historia Natural. En este estudio se presentan los resultados de los análisis paleoparasitológicos realizados en el año 2003 en una muestra de músculo esquelético de la momia. Las muestras fueron fijadas en formol $(\mathrm{pH} 7,2)$ y procesadas por histología de rutina para tinciones de hematoxilina-eosina e inmunohistoquímica de fluorescencia indirecta en secciones de $5 \mu \mathrm{m}$ de espesor. El tejido muscular se encuentra en óptimas condiciones de conservación. Sin embargo, se observó la presencia de estructuras quísticas. A través de inmunofluorescencia indirecta (IFI) con suero humano hiperinmune para Trichinella sp. se demostró que los quistes corresponden a la presencia de triquinosis. Adicionalmente, este hallazgo fue corroborado por especialistas parasitólogos de extensa trayectoria en parasitología pediátrica. Finalmente, es difícil explicar y argumentar la presencia de esta parasitosis en aquella época y en nuestro continente, por lo que este hallazgo ha constituido un verdadero hito dentro de la paleoparasitología mundial.
\end{abstract}

Palabras claves: triquinosis, momia, Cerro El Plomo, trichinellosis, Trichinella, paleoparasitología, Chile.

The Cerro El Plomo mummy is an Inca (1,500 AD) child of about 8 to 9 years old. The boy was found near the top of Cerro El Plomo, Santiago, in the year 1954 and is now under the care of the Museo Nacional de Historia Natural, Chile. In September 2003, as part of a larger study, we examined muscle tissue samples of this mummy. Samples were fixed in formol (pH 7.2) and processed by routine histological techniques, obtaining 5 m thick-sections that were stained with hematoxylin-eosin and processed for immunohistochemical analysis. The histological examination revealed the presence of cystic structures with capsules. Through indirect immunofluorescence (IFI) with human hyperimmune serum to Trichinella sp. the presence of trichinosis was demonstrated. This conclusion was later confirmed by parasitologists with extensive experience in pediatric parasitology. Finally, although it is difficult to explain the presence of this parasite in our continent in pre-Columbian times, this finding is a real milestone in world paleoparasitology.

Key words: Trichinosis, mummy, Cerro El Plomo, trichinellosis, Trichinella, paleoparasitology, Chile.

La paleoparasitología ha permitido reconstruir la historia del parasitismo, y ha aportado información importante sobre la distribución mundial e intercontinental de los parásitos y movimientos humanos. Desde la antigüedad se han desarrollado estudios en restos arqueológicos humanos, coprolitos, sedimentos y tumbas.
La trichinellosis es una zoonosis humana, también conocida como trichinosis o trichiniasis, y popularmente como triquina. Es causada por un grupo de nemátodos del género Trichinella. Esta zoonosis tiene una historia larga y su evidencia en la América precolombina es debatible (Pozio y Murrell 2006).

* Este artículo formó parte del Taller de Bioarqueología "Avances y Desafíos del Paleoambiente y la Paleoparasitología", Arica, noviembre 2008. Parte de sus resultados se publicaron en el volumen 43, número 2 de Chungara, 2011.

1 Unidad de Histoembriología, ICBM, Facultad de Medicina. Universidad de Chile. Avda. Independencia 1027. Santiago, Chile.

* Autor correspondiente: hrodrigu@ med.uchile.cl

2 Hospital Luis Calvo Mackenna, Universidad de Chile, Santiago, Chile. inoemi@med.uchile.cl

3 Departamento de Biología, Universidad de Tarapacá, Arica, Chile. oespinoz@uta.cl

4 Departamento de Morfología, Facultad de Medicina, Universidad del Desarrollo, Santiago, Chile. Subdirección de Museos, DIBAM, Santiago, Chile. mario.castro@museoschile.cl 
La trichinellosis es una infección parasitaria transmitida por carnivorismo. El estado larvario se enquista en el tejido muscular de animales domésticos y salvajes que se alimentan con carne cruda infectada, siendo el cerdo el más afectado al ser alimentado con desechos crudos o en los basurales. La enfermedad se caracteriza por un síndrome febril, signos oculopalpebrales, mialgias, eosinofilia elevada, y hemorragia subungueal. La larva puede sobrevivir por un tiempo largo en la carne en descomposición. En la cadena, la presencia de ratas en las cercanías de la crianza de cerdos juega un rol importante como agentes transmisores y diseminadores de la enfermedad. Los perros y gatos también pueden contagiarse al ingerir carne infectada con larvas de Trichinella spiralis (Gottstein et al. 2009).

Para el hombre moderno una de las fuentes de infección es la ingestión de alimentos de origen porcino (jamones crudos, pancetas ahumadas, embutidos, y eventualmente ceviches). Es importante considerar que la salazón y el ahumado son insuficientes para matar al estado larvario enquistado de la triquina (USDA 2008). Sin embargo, ellos se destruyen por la congelación a $-15{ }^{\circ} \mathrm{C}$ durante 20 días, o a $-30{ }^{\circ} \mathrm{C}$ durante veinticuatro horas (Malakauskas y Kapel 2003). En Europa las carnes de cerdos, zorros, caballos y suinos silvestres han sido identificadas como fuente de infección para el ser humano, y la especie diagnosticada siempre fue Trichinella britovi. Sin embargo, en otras áreas del continente europeo como Suiza, tanto los cerdos domésticos como los silvestres, siempre han sido considerados libres de Trichinella (Muller et al. 2006), indicando que el parásito no es endémico de la región. En general, la infección humana proviene de la ingestión de carnes de animales domésticos y silvestres, aves, peces, especies de abasto, y otras (Gottstein et al. 2009).

Las larvas profundizan en la mucosa intestinal, penetran a través de los capilares linfáticos y venosos y llegan a la circulación general diseminándose por todo el organismo, pero enquistándose en la musculatura estriada esquelética. Las larvas se localizan en el interior de las fibras musculares, las que se destruyen parcialmente; al cabo de quince días quedan rodeadas por una envoltura constituida por el sarcolema (Muller et al. 2006), o depósitos de colágeno (Krivokapich et al. 2006). Así se origina el quiste larval, que mide entre 250 a $400 \mu \mathrm{m}$. Luego de 20 a 30 días, las larvas completan su encapsulamiento generando una fuerte reacción tisular de la fibra muscular y del tejido conectivo circundante, endomisio y perimisio (Krivokapich et al. 2008), y a los seis meses comienza a observarse el depósito de calcio en las paredes del quiste.

Para conocer la naturaleza de la trichinellosis existen referencias desde la antigüedad sobre patologías asociadas o relacionadas a lo que hoy se conoce de las enfermedades semejantes (Ajtar 2003).

En Chile, solo aparecen como relatos clínicos y asociaciones en la Revista Médica de Chile (Actas de reuniones médicas históricas, no catalogadas en la Biblioteca de la Facultad de Medicina de la Universidad de Chile) de fines del 1800 y primera mitad del 1900, siempre con asociación a los inmigrantes alemanes y sus costumbres de fabricar y consumir embutidos. Sin embargo, en la actualidad el parásito es de distribución intercontinental, y el único lugar donde no se ha encontrado es en la Antártica (Pozio 2005; Schenone et al. 2002).

El presente trabajo tiene por objetivo analizar la musculatura estriada de la momia Inca denominada "Niño del Cerro El Plomo", encontrada a 5.200$5.400 \mathrm{msm}$, en las cercanías de Santiago de Chile. Desde 1954 esta momia se encuentra depositada en el Museo Nacional de Historia Natural de Santiago, y en el año 2003 fue llevada al Hospital Clínico de la Universidad de Chile donde se realizó este estudio médico multidisciplinario.

\section{Materiales y Métodos}

Los orígenes de la cultura Inca no están claros. La evidencia arqueológica disponible señala que los Incas fueron influenciados por los imperios Tiwanaku y Wari, los cuales les precedieron. Su economía se basó en la agricultura, la ganadería y practicaron la explotación de minerales. El dominio Inca abarcaba desde el norte de Ecuador hasta Chile central; tenía una longitud de 4.800 kilómetros y un ancho de 460 kilómetros. Su capital era Cuzco, que para la época de la llegada de los españoles tenía unos 100.000 habitantes. Según estudios de la indumentaria y ajuar funerario realizados por especialistas del Museo Nacional de Historia Natural de Santiago, la momia del Cerro El Plomo correspondería a un niño de la cultura Inca, el que alcanzó la región del Mapocho posiblemente hacia el año 1500 d.C., fecha que coincide, según la historia, con la expansión del Reino Inca.

Probablemente, el Niño del Cerro El Plomo fue ofrecido en sacrificio a los dioses cuando 
sólo tenía unos 8 a 9 años de edad, ésta calculada según el grado de desarrollo de las piezas dentarias (Sanhueza et al. 2005). Al momento del hallazgo, se encontraron entre otros utensilios, mocasines de cuero, ajuares y finas ofrendas de plata entre las que destacan dos llamas y una estatuilla femenina ricamente ataviada (Cabezas 1986). El niño fue congelado en forma natural (momificado) y así permaneció hasta el año 1954. Actualmente, se encuentra en el Museo Nacional de Historia Natural de Santiago de Chile, y es mantenido en condiciones de absoluta oscuridad, a $0{ }^{\circ} \mathrm{C}$ y con una humedad del 50\%. El cuerpo se encuentra en buen estado de conservación de los tejidos.

Durante el año 2003, y por iniciativa del Prof. Mario Castro, académico en ese entonces de la Facultad de Medicina de la Universidad de Chile, se reunió a un grupo de investigadores con el propósito de aprovechar un traslado temporal de la momia al Centro de Imagenología del Hospital Clínico de la Universidad de Chile.

El traslado se realizó bajo estrictas condiciones de refrigeración y custodia policial. En la unidad de imágenes cada investigador usó mascarilla y guantes, y mediante un trocar de acero inoxidable quirúrgico de $0,3 \mathrm{~mm}$ de diámetro se obtuvieron tres muestras de la musculatura estriada del músculo vasto interno del cuádriceps derecho.

Las muestras fueron inmediatamente fijadas en formol tamponado $\mathrm{pH} 7,2$, y mantenidas ahí por 96 horas. Luego fueron procesadas por técnicas histológicas de rutina (Venturello et al. 2007) hasta la obtención de secciones de $5 \mu \mathrm{m}$ de espesor montadas en portaobjetos previamente silanizados.
Posteriormente se desarrollaron las tinciones de Hematoxilina y Eosina (H\&E) y la inmunofluorescencia indirecta (IFI) (Choy et al. 1991) con suero hiperinmune (obtenido de niños altamente infectados) incubado con isotiocionato (FITC) para ser observado al microscopio de fluorescencia.

\section{Resultados}

\section{Descripción general}

El Niño del Cerro El Plomo corresponde a un cuerpo momificado, de consistencia extremadamente dura y pétrea. Se encuentra en posición sentada, con las piernas cruzadas y rodillas en alto donde apoya sus brazos cruzados y la cabeza. El cabello es de color negro y grueso y se encuentra pulcramente ordenado y trabajado en trenzas finas, abundantes y muy regulares, dirigidas hacia atrás y abajo. Tiene una constitución ectomórfica y rasgos mongoloides, y antropometría infantil con muy buen estado de conservación estructural.

\section{Descripción de la histología y tinción de Hematoxilina-eosina (H\&E) (Figuras 1 y 2)}

En las secciones histológicas teñidas con H\&E, se observan las fibras musculares estriadas esqueléticas fuertemente acidófilas y estructuralmente bien conservadas, como también el endomisio y perimisio. Sin embargo, no se observan estructuralmente vasos sanguíneos ni linfáticos. Luego y similar a lo descrito en los textos clásicos de parasitología, se observan estructuras de apariencia quística y
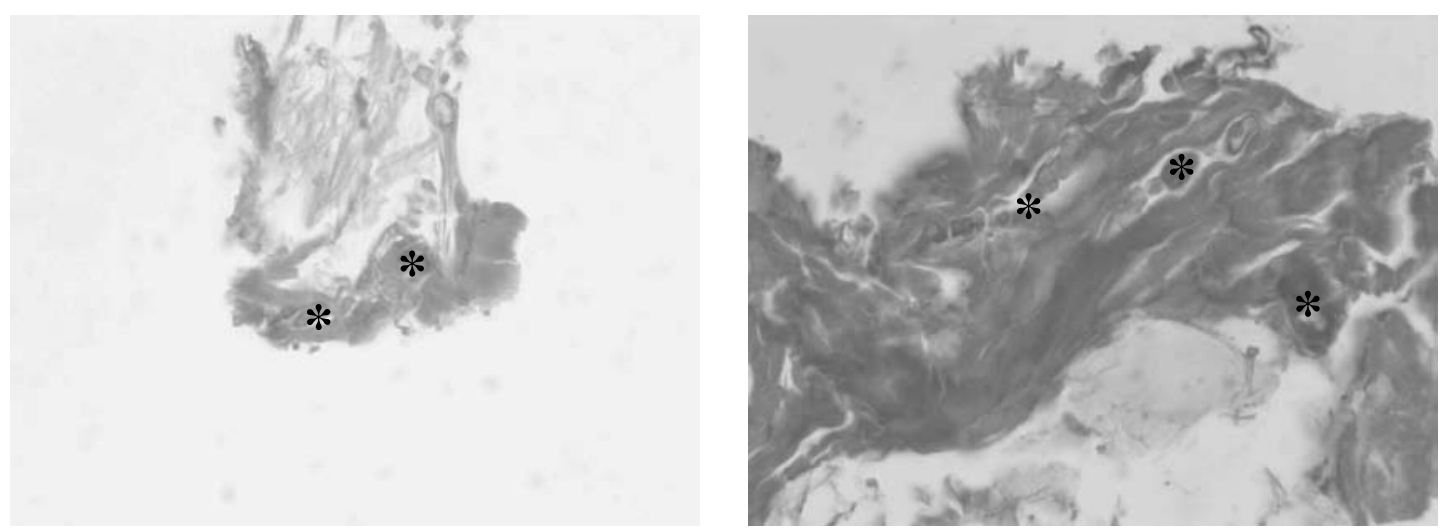

Figuras 1 y 2. Sección histológica de la musculatura estriada esquelética del Niño del Cerro El Plomo, con tinción de H\&E, 40x. (*) indica los quistes de Trichinella sp.

Histological section of striated skeletal muscle tissue of the mummy of Cerro El Plomo, stained with H \& E, 40x. (*) Indicates cysts of Trichinella $s p$. 
aspecto fusiforme/alargado con forma de limón que en su interior presentan uno o varios elementos enrollados de medidas entre $250-500 \mathrm{~mm}$ de diámetro, semejante a lo descrito por López et al. (1992), dando el aspecto morfológico de enquistaciones de Trichinella en la musculatura estriada esquelética del músculo vasto lateral del cuádriceps derecho. Estas enquistaciones son abundantes y de distribución regularmente aisladas y/o con menos frecuencia en agrupaciones. Paralelamente, las enquistaciones presentan un escaso depósito de calcio en la periferia de la estructura quística.

\section{Descripción de la inmunofluorescencia indi- recta (IFI, Figuras 3 y 4)}

Mediante el estudio de microscopia de fluorescencia fue posible observar áreas específicas, bien delimitadas y altamente fluorescentes. Esta observación indica y significa que la reacción es positiva a la presencia de quistes de Trichinella en la musculatura estriada esquelética del Niño del Cerro El Plomo. Paralelamente, con la misma muestra utilizada para IFI, se observó mediante microscopia óptica normal que las áreas fluorescentes coincidían con las enquistaciones descritas anteriormente en la tinción de H\&E.

\section{Discusión}

Las relaciones sociales del hombre entre sí y con animales llevaron al establecimiento de parásitos y enfermedades que algunos de estos ocasionan en el hombre. Luego, la búsqueda de respuestas sobre la presencia de ciertos parásitos en el Nuevo Mundo ha llevado a la formulación de nuevas teorías sobre el poblamiento de América, aunque la presencia de estos parásitos en muestras prehispánicas ha generado una gran controversia (Guhl et al. 2009).

La presencia de los quistes de triquina indica que el Niño del Cerro El Plomo, al momento de su muerte, se encontraba fuertemente afectado por esta parasitosis. Epidemiológicamente es importante destacar las variables de segregación o separación del individuo enfermo, distancia desde los centros poblados, altura, temperatura, biodiversidad de animales terrestres (presencia de rapaces y rapiñas), aéreos, etc., todos los conceptos que en la actualidad se usan efectivamente como elementos importantes en el análisis para la elaboración de los programas de control de ésta y de otras parasitosis.

Paralelamente, Sanhueza et al. (2005) agregan que en el estudio imagenológico del cuerpo llama la atención la presencia de imágenes múltiples de linfonodos axilares que hipotéticamente podrían estar en relación a la presencia de alguna enfermedad infecciosa o parasitaria. La gran pregunta a resolver e investigar es cómo ocurrió la contaminación, dado que según registros epidemiológicos y de fauna silvestre la Trichinella sp. no es propia de América del Sur.

En las dos últimas décadas la epidemiología de la triquinosis ha experimentado importantes avances, destacando especialmente la participación de los ciclos selváticos y de las especies selváticas.
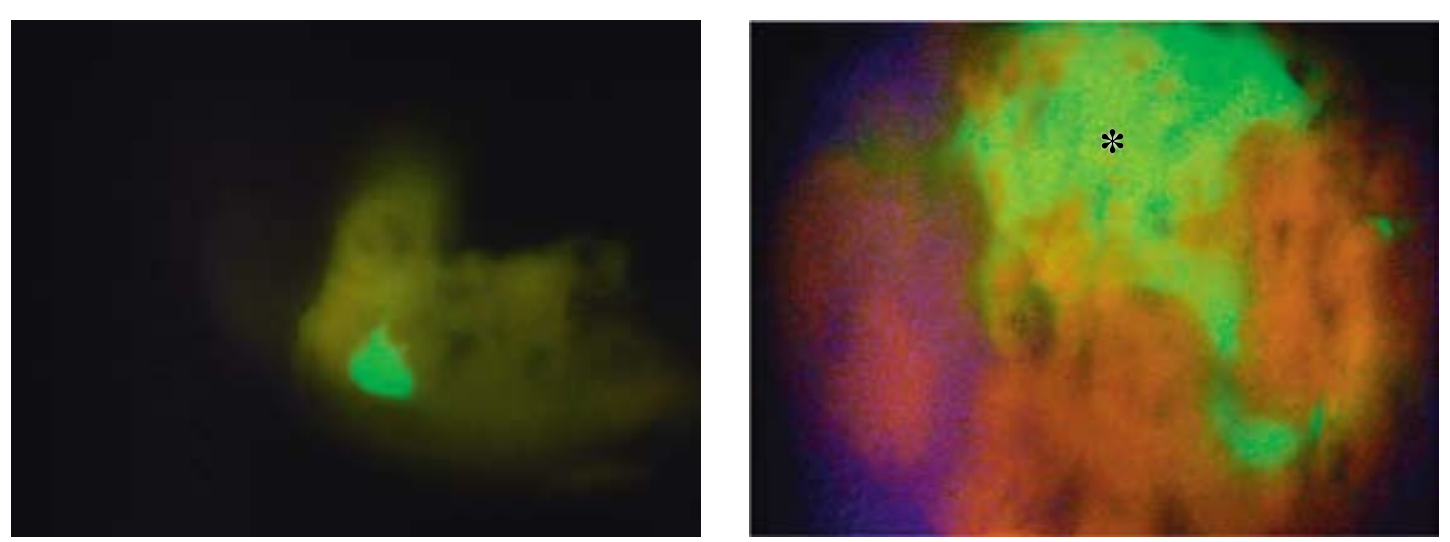

Figuras 3 y 4. Reacción de inmunofluorescencia indirecta (IFI) en sección histológica de la musculatura estriada esquelética del Niño del Cerro El Plomo, con suero hiperinmune contra Trichinella sp. (*) indica los quistes de Trichinella sp. (40x) (fluorescencia verde).

Indirect immunofluorescence (IIF) reaction in histological section of striated skeletal muscle tissue of the mummy of Cerro El Plomo, with hyperimmune serum against Trichinella sp. (*) Indicates cysts of Trichinella sp. 40x (green fluorescence). 
Generalmente Trichinella spiralis se encuentra en el cerdo doméstico, aunque en el último tiempo también se han encontrado otras especies de Trichinella infestando huéspedes como caballos, perros y cerdos salvajes (Pozio y Murrell 2006).

En el mundo, el caso más temprano del que se tiene registro involucra a un joven egipcio que vivía a orillas del río Nilo alrededor del año 1.200 d.C. (Campbell 1983). La información contemporánea comienza a partir de 1835, con James Paget and Richard Owen en Londres, Inglaterra (Owen et al. 2005). A fines del 1800 y con la muerte de Friedrich Zenker en 1860 por Trichinella, en Europa y principalmente en Alemania, se desarrollaron intensos programas de control, aun considerando que la infectividad del nemátodo podría tener variaciones geográficas (Nelson et al. 2003), no obstante se postula que las cepas aisladas de animales silvestres parecen ser de muy baja infectividad para cerdos y ratas, los principales huéspedes modernos (Nelson et al. 1966).

Generalmente se reconocen once genotipos o especies en el género de Trichinella, ocho encapsulados (Figura 2) (T. spiralis, T. Nativa, T. britovi, T. murrelli, T. T6, T. nelsoni, T. T8 y T. T9) y tres no encapsulados (T. pseudospiralis, T. papuae y T. zimbabwensis). Sin embargo, en Europa solo tres especies de Trichinella son más frecuentemente diagnosticadas en carnívoros silvestres ( $T$. britovi, T. nativa y T. pseudospiralis) (Hurnikova y Dvoroznakova 2007; Pozio 2000).

En la distribución geográfica y la relación huésped/parásito, la infección por Trichinella ha sido descrita en todos los continentes a excepción de la Antártica y en un amplio rango de huéspedes (Pozio 2005). En los ciclos domésticos, típicamente se ha diagnosticado $T$. spiralis, aunque también puede invadir hábitats selváticos. El resto de los genotipos son transmitidos y mantenidos en el ciclo selvático, aunque también han sido encontrados en hábitats domésticos y restringidos a ciertas áreas zoogeográficas (Pozio 2000).

Las especies de Trichinella aparentemente se establecieron en América del Norte desde el Mioceno; y probablemente las actividades humanas post colonización permitieron la introducción de T. spiralis en América del Sur, considerando que ninguna otra especie de Trichinella ha sido identificada en los huéspedes selváticos de Sudamérica (Krivokapich et al. 2008; Krivokapich et al. 2006; Larrieu et al. 2004; Pozio 2000).
Por lo tanto, cualquiera sea la especie que pudiera haber infectado al pueblo Inca en el periodo del año 1.500 d.C. es difícil que corresponda a T. spiralis, y por tanto no serían las embarcaciones españolas las responsables de la presencia de este parásito en América del Sur y menos de la región de Chile. Sin embargo, sí puede ser posible que las embarcaciones y expediciones chinas, antes de los españoles, hacia el año 1.000 d.C., pudieran ser las responsables de la diseminación de este y otros parásitos en el continente americano (Taboada 2007).

Ahora, sería importante que a partir de las secciones histológicas de la musculatura esquelética del Niño del Cerro El Plomo se desarrollaran pruebas moleculares para determinar la tipificación antigénica e identificar el tipo de Trichinella encontrada en este niño del pueblo Inca.

Los primeros estudios publicados sobre parásitos zoonóticos fueron por Szidat (1944) quien encuentra huevos de Diphyllobothrium latum en material de Austria fechado en el 1.500 a.C., seguido por los resultados de Dicrocoelium sp. en material de Inglaterra fechado en el 1.100 d.C. (Taylor 1955). En Holanda, Jansen y Boersema (1972) encuentran D. latum, Taenia sp. y huevos de Fasciola hepática en restos del 100 a.C. y 500 d.C. Los resultados paleoparasitológicos desde entonces siempre han mostrado la presencia de parásitos zoonóticos transmitidos principalmente por el consumo y/o el contacto con animales domésticos.

En América del Sur una zoonosis que se sabe que ha ocurrido en una población prehistórica, y que todavía existe en las poblaciones actuales en el Perú y Chile es la presencia de Diphyllobothrium pacificum. Baer (1969) identificó en Perú en pacientes humanos la especie $D$. pacificum, un parásito del lobo marino cuyas larvas contaminan los peces de agua salada y los mariscos, principalmente por el consumo de ceviche, un plato tradicional de las poblaciones costeras del Pacífico elaborado con pescados de mar crudos.

Por otro lado, en el contexto histórico, la dominación Inca en la región de Santiago se inició hacia el 1483, antes de la caída de los centros del imperio Inca en 1533, bajo el mandato de Tupa Inca Yupanki. A este período correspondería el Niño del Cerro El Plomo (Barbín 1984), donde la datación relativa está basada en los estudios del ajuar funerario del niño (MNHN 1957), y apoyado por el contexto histórico Inca, la arquitectura del santuario y los datos etnoarqueológicos (Cabezas 1986). 
La identificación de aquellas zoonosis que afectaban a los pueblos ancestrales, la comprensión y análisis de los mecanismos de transmisión y los factores que afectaron esta dinámica biogeográfica, ecológica, cultural, histórica o social, pueden aportar al estudio epidemiológico de las culturas antiguas y con ello aportar a las metodologías modernas.

Existen algunas figuras históricas que han sido asociadas a la triquinosis. Hirschmann (2001) analizó la historia y sintomatología que llevó a la muerte a Wolfgang Amadeus Mozart, concluyendo como causa de muerte una infestación con Trichinella al consumir carne de cerdo. Dorozynski (1998) reportó la presencia de Trichinella en el castillo y casas de campo que rodea a Marly-le-Roi, cerca de París, lugar de caza de Luis XIV de Francia, quien llegó al trono a la edad de 5 años y reinó durante 72 años desde 1643 hasta 1715 . Adicionalmente, en el periodo Inca no existían los suinos en América e incluso en España.

En relación al cerdo, el género originario de todos los porcinos del mundo es el Sus; sólo en algunos casos se admite la existencia del subgénero, e incluso con distintas denominaciones. Se asegura la existencia de tres subgéneros: mediterraneus, de origen africano y extendido por las regiones del sur de Europa; el Ferus o cerdo salvaje extendido por toda Europa, y el Stratosus, o cerdo de corbata, más pequeño que los anteriores y de origen asiático. Laguna (1989, citado por Aparicio y Vargas 2003) considera las tres denominaciones sin atribuirle la clasificación de subgénero y abordando dos opciones acerca del origen del Mediterraneus, en transición entre los otros dos o descendientes de la domesticación del Scrofa ferus. Mientras que otros contemplan tres especies: Sus Scrofa, Sus Mediterraneus y Sus Vittatus, de las que procederían respectivamente los troncos céltico, ibérico y asiático. Se admite la posibilidad de que el Sus Mediterraneus fuera una subespecie del Sus Scrofa, teoría que comparte Aparicio (1988, citado por Aparicio y Vargas 2003), al admitir que el ibérico sería uno de los tipos representativos del Sus scrofa mediterraneus. Sólo Aparicio (1988, citado por Aparicio y Vargas 2003), considera a mediterraneus como una subespecie de scrofa; el resto de los autores obvian la subespecie y establecen como los tres troncos prehistóricos los siguientes: Sus scrofa (jabalí europeo), Sus mediterraneus (jabalí mediterráneo) y Sus vittatus (cerdo asiático) (Aparicio y Vargas 2003).
A Sudamérica el jabalí ingresa a través de Argentina hacia el año 1906 (Jackson 1989). Los jabalíes son nativos de gran parte de Europa del Norte y Central, la región mediterránea (incluyendo norte de África) y gran parte de Asia hasta el sur de Indonesia. Las poblaciones también se han introducido artificialmente en otras partes del mundo, en particular las Américas y Australasia, principalmente para la caza. La importación a tierra americana fue a mediados del siglo XVI por Hernán Cortés y Hernando de Soto, y a mediados del siglo XVII por La Salle.

En la actualidad, los mamíferos marinos, caribú, y las aves migratorias son reservorios de infección parasitaria para humanos (Reinhard 1990). La trichinellosis como zoonosis está ampliamente distribuida y adaptada a los distintos tipos de clima y regiones del mundo. A causa de su baja especificidad para reproducirse y mantenerse en todos los ambientes, es una parasitosis que afecta a la mayoría de los mamíferos, aunque también se la ha observado en aves, reptiles y artrópodos (Kapel 2000). Aunque Trichinella sp. es un parásito intracelular extremadamente lábil a las bajas temperaturas (Lee y Shivers 1987).

En relación a la aparición de la enfermedad de trichinellosis esta fue de modo contemporáneo tanto en Chile como en Argentina. Aparecen en la literatura datos sobre las primeras sospechas sintomatológicas consistentes con el diagnóstico de triquinosis en humano hacia 1898 (Ribicich et al. 2005).

La primera demostración científica de Trichinella sp. en América Latina fue en 1863, a partir de un cerdo transado en Valparaíso-Chile por la tripulación de un barco alemán. El parásito fue identificado en el tejido muscular del animal y en uno de los marineros muertos con síntomas y signos típicos de trichinellosis (Gould 1970). Mientras que en Paraguay y Brasil no se registran reportes de trichinellosis en humanos y animales (Steffan 2006).

En las lesiones quísticas de triquinosis se describe una reacción inflamatoria importante con calcificación periférica del tejido del huésped, ya observable al triquinoscopio (Gamito-Santos et al. 2009).

Desde una perspectiva evolutiva, es posible la utilización de los parásitos como marcadores biológicos, permitiendo un nuevo enfoque sobre las migraciones humanas y colonización de continentes. La dispersión y distribución de los parásitos en el tiempo puede ser utilizada para 
rastrear las migraciones de sus huéspedes humanos (Gonçalves et al. 2003). Paralelamente, la identificación de aquellas zoonosis que afectaban a los pueblos ancestrales, la comprensión y análisis de los mecanismos de transmisión y los factores que afectaron esta dinámica biogeográfica, ecológica, cultural, histórica y social pueden aportar al estudio epidemiológico de las culturas antiguas y con ello aportar a las metodologías modernas.

Finalmente, es posible que la presencia de estos parásitos sea una evidencia del contacto transpacífico entre América y las culturas asiáticas, aún siendo posible la teoría de la hipobiosis (estado durmiente por largos periodos).

\section{Conclusiones}

La momia conocida como Niño del Cerro El Plomo, al momento de su muerte se encontraba altamente infestada con Trichinella sp. Simultáneamente, es posible afirmar que Trichinella sp. existía en América del Sur desde antes de la llegada de los españoles.

Agradecimientos: Agradecimientos sinceros al Prof. Mario Castro y a la Dirección del Museo Nacional de Historia Natural, por habernos dado la hermosa oportunidad de conocer al Niño del Cerro El Plomo y estudiar los fantásticos hallazgos obtenidos. Como también por el esmero de los cuidados de preservación que desarrollan en el cuidado de las valiosas piezas Incas. También al Sr. Juan Borja, Ayudante Técnico de Investigación de la Unidad de Inmunohistoquímica del Programa de Anatomía y Biología del Desarrollo de la Facultad de Medicina de la Universidad de Chile; y al convenio de Desempeño UTA-MINEDUC 2008-2010 $\mathrm{y}$ al proyecto FONDECYT 1101046.

\section{Referencias Citadas}

Ajtar, S. 2003. Por qué los musulmanes no comen carne de cerdo. http://www.revistaarabe.com.ar/etica_07.asp. (consultada el 4 de mayo del 2009).

Aparicio, M.A. y J.D. Vargas 2003. El cerdo Ibérico y el bienestar animal. Ganadería 23:30-35.

Baer, J. 1969. Diphyllobothrium pacificum, a tapeworm from sea lions endemic in man along the coastal area of Peru. Journal Fish Research Board Canada 26:717-723.

Barbín, C. 1984. Los hallazgos de la paleopatología. Creces 5:81-82.

Cabezas, A. 1986. El Santuario de Altura Inca Cerro El Plomo. Tesis de Grado para optar al título de Licenciado en Arqueología y Prehistoria de Chile, Universidad de Chile, Santiago.

Campbell, W. 1983. Historical introduction. En Trichinella and Trichinosis, editado por W.C. Campbell, pp. 1-30. Plenum Press, New York and London.

Choy, W., M. Ng y P. Lim 1991. Trichinella spiralis: Light microscope monoclonal antibody localization and immunochemical characterization of phosphorylcholine and other antigens in the muscle larva. Experimental Parasitology 73:172-183.

Dorozynski, A. 1998. Parasites likely to have plagued Louis XIV. British Medical Journal 316:1477.

Gamito-Santos, J., L. Gómez, R. Calero-Bernal, J. Rol-Díaz, L. González-Ruibal, M. Gómez-Blázquez y J. Pérez-Martín 2009. Histopathology of trichinellosis in wild boar. Veterinary Parasitology 165:165-169.

Gonçalves, M., A. Araújo y L. Ferreira 2003. Human intestinal parasites in the past: New findings and a review. Memórias do Instituto Oswaldo Cruz 98 (Suppl. I):103-118.
Gottstein, B., E. Pozio y K. Nockler 2009. Epidemiology, diagnosis, treatment, and control of trichinellosis. Clinical Microbiology Reviews 22:127-145.

Gould, S.E. 1970. Trichinosis in Man and Animals. C.C. Thomas, Florida.

Guhl, F., M. Ortiz, A. Moncayo y A. Patiño 2009. Parásitos y evolución. http://ciencias.uniandes.edu.co/pdf/parasitos.pdf. (consultada el 06 de mayo del 2009).

Hirschmann, J. 2001. What killed Mozart? Archives International Medicine 161:1381-1389.

Hurnikova, Z. y E. Dvoroznakova 2007. Long-term survey on trichinellosis in wildlife of Slovakia. XII International Conference on Trichinellosis. Book of Abstracts, pp. 63. National Park Plitvice Lakes, Croatia.

Jackson, J. 1989. Jabalíes y chanchos salvajes. Presencia 3:8-10.

Jansen, J. y J. Boersema 1972. Helminth eggs from the latrines of the Olofskapel Gatehouse, Amsterdam. Paleopathology Newsletter 2:ab7-ab8.

Kapel, C. 2000. Host diversity and biological characteristics of the Trichiniella genotypes and their effect on transmission. Veterinary Parasitology 93:293-307.

Krivokapich, S., C. González Prous, G. Gatti, V. Confalonieri, V. Molina, H. Matarasso y E. Guarnera 2008. Molecular evidence for a novel encapsulated genotype of Trichinella from Patagonia, Argentina. Veterinary Parasitology 156:234-240.

Krivokapich, S., V. Molina, H. Bergagna y E. Guarnera 2006. Epidemiological survey of Trichinella infection in domestic, synanthropic and sylvatic animals from Argentina. Journal of Helminthology 80:267-269. 
Larrieu, E., V. Molina, S. Albarracín, S. Mancini, R. Bigatti, L. Ledesma, C. Chiosso, S. Krivokapich, E. Herrero y E. Guarnera 2004. Porcine and rodent infection with Trichinella, in the Sierra Grande area of Río Negro Province, Argentina. Annals of Tropical Medicine and Parasitology 98:725-731.

Lee, D. y R. Shivers 1987. A freeze-fracture study of muscle fibres infected with Trichinella spiralis. Tissue Cell 19:665-671.

López, R., C. Muñoz, C. Nazar, A. Rojas y H. Schenone 1992. Prevalencia actual de la triquinosis en Santiago, Chile (1992). Estudio en 500 cadáveres. Boletín Chileno de Parasitología 47:86-87.

Malakauskas, A. y C. Kapel 2003. Tolerance to low temperatures of domestic and sylvatic Trichinella sp. in rat muscle tissue. Journal of Parasitology 89:744-748.

MNHN 1957. La momia del Cerro El Plomo. Boletín del Museo Nacional de Historia Natural 27:31-81.

Muller, N., H. Sager, M. Schuppers y B. Gottstein 2006. Methods for investigating Trichinella infections in domestic and wild animals. Schweizer Archiv für Tierheilkunde 148:463-471.

Nelson, G., E. Blackie y J. Mukundi 1966. Comparative studies on geographical strains of Trichinella spiralis. Transactions of the Royal Society of Tropical Medicine and Hygiene 60:471-480.

Nelson, M., T. Wright, A. Pierce y R. Krogwold 2003. A commonsource outbreak of trichinosis from consumption of bear meat. Journal of Environmental Health 65:16-24.

Owen, I., M. Gómez Morales, P. Pezzotti y E. Pozio 2005. Trichinella infection in a hunting population of Papua New Guinea suggests an ancient relationship of Trichinella with human beings. Transactions of the Royal Society of Tropical Medicine and Hygiene 99:618-624.

Pozio, E. 2000. Factors affecting the flow among domestic, synanthropic and sylvatic cycles of Trichinella. Veterinary Parasitology 93:241-262.

- - - - 2005. The broad spectrum of Trichinella host: from cold-to warm-blooded animals. Veterinary Parasitology 132:3-11.
Pozio, E. y K. Murrell 2006. Systematics and epidemiology of Trichinella. Advances in Parasitology 63:367-439.

Reinhard, K. 1990. Archaeoparasitology in North America. American Journal Physical Anthropology 82:145-163.

Ribicich, M., H. Gamble, A. Rosa, J. Bolpe y A. Franco 2005. Trichinellosis in Argentina: An historical review. Veterinary Parasitology 132:137-142.

Sanhueza, A., L. Pérez, J. Díaz, D. Busel, M. Castro y A. Pierola 2005. Paleoradiología: estudio imagenológico del niño del cerro El Plomo. Revista Chilena de Radiología 11:184-190.

Schenone, H.F., A. Olea, H. Schenone, M. Contreras, R. Mercado, L. Sandoval y C. Pavletic 2002. Situación epidemiológica actual de la triquinosis en Chile. 1991-2000. Revista Médica de Chile 130:281-285.

Steffan, P. 2006. Trichinellosis en el Cono Sur de América: situación actual y prospectiva de una zoonosis parasitaria ancestral. Red de Helmintología de FAO para América Latina y el Caribe. http://www.produccion-animal.com.ar/sanidad_intoxicaciones_metabolicos/parasitarias/parasitarias_cerdos/01-trichinellosis. pdf. (Consultada el 01 de julio del 2011).

Szidat, L. 1944. Über die Erhaltungsfähigkeit von Helmintheneiern in Vor- und Frühgeschichtlichen Moorleichen. Z Parasitenkd 13:265-274.

Taboada, H.G.H. 2007. Reseña de "1421: el año en que China descubrió el mundo" de Gavin Menzies. Traducido por F.J.R. Mena. Estudios de Asia y Africa 42:760-769.

Taylor, E. 1955. Parasitic helminths in medieval remains. Veterinary Record 67:218-228.

USDA 2008. Jamón y la inocuidad alimentaria. http://mailing. netcommerce.com.mx safefood/safefood/safefood/18_100.htm. (04 de mayo del 2009).

Venturello, S., S. Verzoletti, N. Costantino, M. Forastiero y M. Roux 2007. Early pulmonary response in rats infected with Trichinella spiralis. Parasitology 134:281-288. 